\title{
Some Classical and Quantum Algebras
}

\author{
Bong H. Lian and Gregg J. Zuckerman
}

\begin{abstract}
We discuss the notion of a Batalin-Vilkovisky (BV) algebra and give several classical examples from differential geometry and Lie theory. We introduce the notion of a quantum operator algebra (QOA) as a generalization of a classical operator algebra. In some examples, we view a QOA as a deformation of a commutative algebra. We then review the notion of a vertex operator algebra (VOA) and show that a vertex operator algebra is a QOA with some additional structures. Finally, we establish a connection between BV algebras and VOAs.
\end{abstract}

\section{Introduction}

In reference [19], the authors established a precise and general connection between two types of algebras which are well known in contemporary mathematical physics: Batalin-Vilkovisky algebras (BV algebras) and vertex operator algebras (VOAs). BV algebras, although implicit in modern mathematics, arose for the first time explicitly in the context of BV quantization of classical field theories. VOAs arose for the first time in the parallel contexts of two dimensional conformal quantum field theory [1] and the mathematical theory of monstrous moonshine [2] [5].

The present paper may be regarded as a very brief mathematical introduction to both BV algebras and VOAs. We have attempted to relate both types of algebras to objects and constructions in differential geometry, supergeometry, Lie theory, commutative algebra, homological algebra and operator algebras.

In section 2, we point out that the algebra of differential forms on a semiRiemannian manifold is naturally a BV algebra. Thus, BV algebras were implicit long ago in the absolute tensor calculus and in general relativity. Likewise, the algebra of exterior forms on a finite dimensional Lie algebra, which is endowed with a nondegenerate symmetric bilinear form (or Hermitian form), is naturally a BV algebra.

As a pedagogical device in our discussion of VOAs, we introduce in section 3 the very simple and abstract notions of quantum operators and quantum operator algebras (QOAs). Physicists have shied away from such abstractions, but we have no such inhibitions. We have found that many of the fundamental

\footnotetext{
${ }^{0} 1991$ Mathematics Subject Classification. Primary 81T70, 17B68.

${ }^{0}$ G.J.Z. is supported by NSF Grant DMS-9008459 and DOE Grant DE-FG0292ER25121.
} 
ideas and formulas in conformal field theory can be very naturally explained in the setting of QOAs.

In order to connect QOAs to VOAs, we present in section 1 a quick discussion of three points of view on commutative algebras. We then review the definition of a VOA in section , and discuss this definition from three new points of view that parallel our previous discussion in section 1 . The main new result of the present paper is Theorem 5.6, which reformulates the notion of a VOA (without a Virasoro quantum operator) as a particular and remarkable type of QOA, which we call a creative QOA. We then present two fundamental constructions of creative QOAs. The second construction is a reformulation of some work of Igor Frenkel and Yongchang Zhu [7]. The approach of the present paper was strongly influenced by the Frenkel-Zhu article.

In the final section of this paper (see Theorems 6.3 and 6.4), we restate the main theorems of [19] as theorems about conformal QOAs (which are now equipped with a Virasoro quantum operator). In brief, we employ the BRST precedure [14] 3] [4] to formulate a cohomological construction of a BV algebra that starts from a choice of conformal QOA with central charge twenty-six. Our main example requires one ingredient: a simple Lie algebra over the complex numbers. Our construction goes through without a hitch because of the remarkable fact that there does not exist a simple Lie algebra having dimension twenty-six.

Many other known constructions in string theory can in fact be formulated in the language of QOAs and the BRST procedure (see also [24] 26] 16] [17] [18]). The results on BV algebras in 19 have been applied in a recent paper by Greg Moore [20].

The present paper grew out of a pair of lectures presented by the authors in October 1993 to the University of North Carolina Mathematics Department. We thank James Stasheff for the opportunity to give these lectures. The authors are very pleased to dedicate this paper to Professor Kostant on the occasion of his sixty-fifth birthday.

\section{Batalin-Vilkovisky Algebras}

Let $A^{*}$ be a $\mathbf{Z}$ graded commutative associative algebra. For every $a \in A$, let $l_{a}$ denote the linear map on $A$ given by the left multiplication by $a$. Recall that a (graded) derivation $d$ on $A$ is a homogeneous linear operator such that $\left[d, l_{a}\right]-l_{d a}=0$ for all $a$. A BV operator [25] [23] 11] $\Delta$ on $A^{*}$ is a linear operator of degree -1 such that:

(i) $\Delta^{2}=0$; 
(ii) $\left[\Delta, l_{a}\right]-l_{\Delta a}$ is a derivation on $A$ for all $a$, ie. $\Delta$ is a second order derivation.

A BV algebra is a pair $(A, \Delta)$ where $A$ is a graded commutative algebra and $\Delta$ is a BV operator on $A$. The following is an elementary but fundamental lemma:

Lemma 2.1 11]2] Given a BV algebra $(A, \Delta)$, define the $B V$ bracket $\{$,$\} on$ A by:

$$
(-1)^{|a|}\{a, b\}=\left[\Delta, l_{a}\right] b-l_{\Delta a} b .
$$

Then $\{$,$\} is a graded Lie bracket on A of degree -1$.

By property (ii) above, it follows immediately that for every $a \in A,\{a,-\}$ is a derivation on $A$. Thus a BV algebra is a sort of odd Poisson algebra which, in mathematics, is also known as a Gerstenhaber algebra [9] 10].

\subsection{Some Classical Examples}

Let $M$ be an $n$-dimensional smooth manifold with a fixed volume form $\omega$. Let $C^{*}(M)$ be the deRham complex. Let $V^{*}(M)$ be the algebra of polyvector fields - ie. the exterior algebra on the smooth vector fields $V e c t(M)$ over the the ring of smooth functions on $M$. There is a canonical degree reversing linear isomorphism $i_{\omega}: V^{*}(M) \longrightarrow C^{n-*}(M)$ given by the contraction with $\omega: v \mapsto$ $i_{\omega}(v)$. Conjugating the deRham differential $d$ by this isomorphism, we obtain a square zero degree -1 operator $\Delta_{\omega}=i_{\omega}^{-1} d i_{\omega}$ on $V^{*}(M)$. In local coordinates $x^{i}$ for which the volume form is $\omega=d x^{1} \wedge \cdots \wedge d x^{n}$, we have

$$
\Delta_{\omega}=\sum_{i} \frac{\partial}{\partial x^{i}} \frac{\partial}{\partial x^{i^{*}}}
$$

where $x^{i^{*}}$ denotes the generator $\frac{\partial}{\partial x^{i}}$ in $V^{1}(M)$. It is evident that $\Delta_{\omega}$ is a second order derivation on $V^{*}(M)$, hence making $\left(V^{*}(M), \Delta_{\omega}\right)$ into a BV algebra [23].

Clearly the above construction generalizes to spaces in other categories: algebraic, holomorphic etc.

The construction may be viewed slightly differently: we can regard $V^{*}(M)$ as the commutative superalgebra of functions on $\Pi T^{*} M$, the cotangent bundle of $M$ with the fibers made into odd supervector spaces. The BV bracket in $V^{*}(M)$ turns out to be equal to the odd Poisson bracket associated to the canonical odd symplectic two-form on $\Pi T^{*} M$. The bracket is also known as the Schouten bracket.

We should mention an important and well-known application of the algebra $V^{*}(M)$. Let $P$ be a bivector field on $M$. We can always construct a bracket 
operation on the function algebra $C^{\infty}(M)$ by the formula

$$
[f, g]_{P}=\iota_{P}(d f \wedge d g)
$$

where $\iota_{P}$ denotes the contraction of $P$ against a two-form. The question is: when does the new bracket [,] give rise to a Lie algebra structure on $C^{\infty}(M)$ (hence a Poisson algebra)?

Proposition 2.2 The bracket [,] satisfies the Jacobi identity iff the BV bracket $\{P, P\}=0$.

To discuss our second example, let's assume that the volume form $\omega$ above comes from a metric $g$ on $M$. Let $d^{*}$ be the formal adjoint of the deRham differential $d$, relative to the metric $g$. Note that $d^{*}$ is a square zero degree -1 operator on $C^{*}(M)$. Choose local coordinates so that the Riemannian volume form is $\omega=d x^{1} \wedge \cdots \wedge d x^{n}$. Then once again $d^{*}$ has exactly the same form (2.1) where now $x^{i^{*}}$ denotes the generator $d x^{i}$ in the algebra $C^{*}(M)$. Therefore $d^{*}$ is also a second order derivation making $\left(C^{*}(M), d^{*}\right)$ into a BV algebra which depends on $g$.

It turns out that this BV algebra is isomorphic to $\left(V^{*}(M), \Delta_{\omega}\right)$. Locally this isomorphism is determined by $d x^{i} \mapsto g^{i j} \frac{\partial}{\partial x^{j}}$. The metric ensures that this is well-defined globally.

We now come to our third example, which arises in Lie theory. Let $\mathfrak{g}$ be any Lie algebra and $\Lambda^{*} \mathfrak{g}$ be its exterior algebra. Let $\delta$ be the Lie algebra homology differential on $\bigwedge^{*} \mathfrak{g}$

$$
\delta\left(X_{1} \wedge \cdots \wedge X_{p}\right)=\sum_{i<j}(-1)^{i+j}\left[X_{i}, X_{j}\right] \wedge X_{1} \wedge \cdots \hat{X}_{i} \cdots \hat{X}_{j} \cdots \wedge X_{p} .
$$

Then by direct computation we verify that $\delta$ is a BV operator on the exterior algebra.

\section{Quantum Operator Algebras}

Let $V$ be a $\mathbf{Z}$ doubly graded vector space $V=\oplus V^{n}[m]$ such that $V[m]=0$ for all but finitely many negative $m$ 's. The degrees of a homogeneous element $v$ in $V^{n}[m]$ will be denoted as $|v|=n,\|v\|=m$ respectively. In physical applications, $|v|$ will be the fermion number of $v$. In conformal field theory, $\|v\|$ will be the conformal dimension of $v$.

Let $z$ be a formal variable with degrees $|z|=0,\|z\|=-1$. Then it makes sense to speak of a homogeneous (biinfinite) formal power series $a(z)=$ 
$\sum_{n \in \mathbf{Z}} a(n) z^{-n-1}$ of degrees $|a(z)|,\|a(z)\|$ where the coefficients $a(n)$ are linear maps in $V$ with degrees $|a(n)|=|a(z)|,|| a(n)||=-n-1+\|a(z)\|$. Note then that the terms $a(n) z^{-n-1}$ indeed have the same degrees $|a(z)|,\|a(z)\|$ for all $n$. We call a finite sum of such homogeneous series $a(z)$ a quantum operator on $V$, and we denote the linear space of quantum operator as $Q O(V)$.

Clearly it does not makes sense in general to multiply two quantum operators $a(z), b(z)$ pointwise. However the space $\operatorname{Lin}(V)$ of graded linear maps in $V$ can be viewed as a subspace of $Q O(V)$ where a linear map $A$ is regarded as the constant series $\sum A \delta_{n,-1} z^{-n-1}$. The space $\operatorname{Lin}(V)$ is an associative algebra in a canonical way. Is there a natural way to extend the product in $\operatorname{Lin}(V)$ to all of $Q O(V)$ ? One such (nonassociative) extension is known as the Wick product, defined as:

$$
: a(z) b(z):=\sum_{n<0} a(n) z^{-n-1} b(z)+(-1)^{|a||b|} b(z) \sum_{n \geq 0} a(n) z^{-n-1} .
$$

It is evident that when restricted to $\operatorname{Lin}(V)$, the Wick product coincides with the natural product in $\operatorname{Lin}(V)$.

Here we introduce a notation for iterated Wick products which we will use later. Let $a_{1}(z), \ldots, a_{n}(z)$ be quantum operators. Their Wick product is defined inductively as follows:

$$
: a_{1}(z) \cdots a_{n}(z): \stackrel{\text { def }}{=}: a_{1}(z)\left(: a_{2}(z) \cdots a_{n}(z):\right):
$$

There is in fact a family of products (of which the Wick product is one) which measure formally the singularity of the formal product $a(z) b(w)$ as $z$ "approaches" $w$.

Definition 3.1 For each integer $n$ we define a product on $Q O(V)$ :

$a(w) \circ_{n} b(w)=\operatorname{Res}_{z} a(z) b(w) \iota_{z, w}(z-w)^{n}-(-1)^{|a||b|} \operatorname{Res}_{z} b(w) a(z) \iota_{w, z}(z-w)^{n}$

where

$$
\begin{aligned}
& \iota_{z, w}(z-w)^{n}=\sum_{i \geq 0}\left(\begin{array}{c}
n \\
i
\end{array}\right)(-1)^{i} z^{n-i} w^{i} \\
& \iota_{w, z}(z-w)^{n}=\sum_{i \geq 0}\left(\begin{array}{c}
n \\
i
\end{array}\right)(-1)^{n-i} z^{i} w^{n-i} .
\end{aligned}
$$

To see that the above products are well-defined, take an element $v$ in $V$ and consider first

$$
\operatorname{Res}_{z} a(z) v \iota_{w, z}(z-w)^{n}=\sum_{i \geq 0}\left(\begin{array}{c}
n \\
i
\end{array}\right)(-1)^{n-i} w^{n-i} a(i) v .
$$


This is a finite sum because $a(i) v=0$ for all but finitely many positive $i$. So $\operatorname{Res}_{z} b(w) a(z) v \iota_{w, z}(z-w)^{n}$ makes sense for any $v$ and hence defines an element in $Q O(V)$. Similarly for $\operatorname{Res}_{z} a(z) b(z) v \iota_{z, w}(z-w)^{n}$. We note that $a(z) \circ_{-1} b(z)$ is nothing but the Wick product $: a(z) b(z):$.

Proposition 3.2 For $a(z), b(z)$ in $Q O(V)$, the following equality of formal power series in two variables holds:

$$
a(z) b(w)=\sum_{n \geq 0} a(w) \circ_{n} b(w) \iota_{z, w}(z-w)^{-n-1}+: a(z) b(w): .
$$

In this sense $: a(z) b(w):$ is the nonsingular part of the operator product expansion (3.9) (see [1]), while $a(w) \circ_{n} b(w) \iota_{z, w}(z-w)^{-n-1}$ is the polar part of order $-n-1$. We note that for $n<0$, we have

$$
a(z) \circ_{n} b(z)=\frac{1}{(-n-1) !}: \partial^{-n-1} a(z) b(z):
$$

where $\partial=\frac{d}{d z}$. For $n \geq 0$, we have

$$
a(z) \circ_{n} b(z)=\left[\left(\sum_{m=0}^{n}\left(\begin{array}{c}
n \\
m
\end{array}\right) a(m)(-z)^{n-m}\right), b(z)\right] .
$$

Lemma 3.3 Let $A$ be a homogeneous linear operator on $V$. Then the commutator $[A,-]$ is a graded derivation of each of the products ${ }_{{ }_{n}}$.

Note that for $n=0$, we have $a(z) \circ_{0} b(z)=[a(0), b(z)]$. As a corollary, we have

Proposition 3.4 For any $a(z), b(z), c(z)$ in $Q O(V)$ and $n$ integer, we have

$$
a(z) \circ_{0}\left(b(z) \circ_{n} c(z)\right)=\left[a(z) \circ_{0} b(z)\right] \circ_{n} c(z)+(-1)^{|a||b|} b(z) \circ_{n}\left[a(z) \circ_{0} c(z)\right],
$$

ie. $a(z) \circ_{0}$ is a derivation of every product in $Q O(V)$.

The products $\circ_{n}$ will become important for describing the algebraic and analytic structures of certain algebras of quantum operators. Thus we introduce the following mathematical definitions:

Definition 3.5 We say that $a(z), b(z)$ are mutually local if $a(z) \circ_{n} b(z)=0$ for all but finitely many positive $n$. 
Definition 3.6 A graded subspace $\mathcal{A}$ of $Q O(V)$ containing the identity operator and closed with respect to all the products $\circ_{n}$ is called a quantum operator algebra. A $Q O A \mathcal{A}$ is called local if its elements are pairwise mutually local.

We observe that for any element $a(z)$ of a QOA, we have $a(z) \circ_{-2} 1=\partial a(z)$. Thus a QOA is closed with respect to formal differentiation.

Proposition 3.7 Suppose $O$ is a local QOA. Then for any $a(z), b(z)$ in $O$, the matrix entries of $a(z) b(w)$ are rational.

Proof: Let $v$ be in $V$ and $v^{*}$ in the restricted dual of $V$. By eqn (3.9), we have

$$
\left\langle v^{*}|a(z) b(w)| v\right\rangle=\sum_{n \geq 0}\left\langle v^{*}\left|a(w) \circ_{n} b(w)\right| v\right\rangle(z-w)^{-n-1}+\left\langle v^{*}|: a(z) b(w):| v\right\rangle .
$$

Each of the matrix entries, $\left\langle v^{*}\left|a(w) \circ_{n} b(w)\right| v\right\rangle$ is a Laurent polynomial in $w$. If $a(z), b(z)$ are mutually local, then we have only finitely many $(z-w)$-polar terms in the above expansion. Thus the singular part of the operator product expansion contributes a rational function of $z, w$ to the matrix entry. The matrix entry of the Wick product is a Laurent polynomial.

We note that none of the products $\circ_{n}$ is associative in general. (We will return to this point later in an example.) However it clearly makes sense to speak of the left, right or two sided ideals in a QOA and they are defined in an obvious way.

We now return to a classical setting to motivate a construction in the theory of QOAs.

\section{Commutative Algebras}

Let $A$ be a vector space with a distinguished nonzero element 1 . We want to consider the set $C(A, \mathbf{1})$ consisting of commutative, associative bilinear products $m$ on $A$ such that $\mathbf{1}$ is the identity element for $m$. We want to relate this set to some other sets naturally associated to the based space $(A, \mathbf{1})$.

Definition 4.1 A translation map for $(A, \mathbf{1})$ is a linear map $Y: A \longrightarrow$ Lin $(A), a \mapsto Y(a)$, such that the following axioms hold:

(1) $Y(\mathbf{1})=i d$

(2) $Y(a) \mathbf{1}=a$ 
(3) $Y(a) Y(b)=Y(b) Y(a)=Y(Y(a) b)$.

Let $T(A, \mathbf{1})$ denote the set of all translation maps for $(A, \mathbf{1})$.

A translation map $Y$ translates states (elements of $A$ ) into operators (elements of $\operatorname{Lin}(A))$. For each translation map, we can define a product $m_{Y}: A \otimes A \longrightarrow A$

$$
m_{Y}(a, b)=Y(a) b .
$$

Then $m_{Y}$ is commutative and associative and $\mathbf{1}$ is the identity element. Moreover the map $Y$ is an injective homomorphism of algebra $\left(A, m_{Y}, \mathbf{1}\right)$ into the algebra $\operatorname{Lin}(A)$.

Conversely if $m \in C(A, \mathbf{1})$, we can define the map $Y_{m}: A \longrightarrow \operatorname{Lin}(A)$

$$
Y_{m}(a) b=m(a, b) .
$$

Then it is clear that $Y_{m}$ is a translation map.

Proposition 4.2 The sets $C(A, \mathbf{1})$ and $T(A, \mathbf{1})$ are in bijective correspondence via the map $m \mapsto Y_{m}$, and its inverse $Y \mapsto m_{Y}$.

\subsection{Creative Operator Algebras}

The above Proposition is of course trivial to prove, but it is a stepping stone to the following idea. Let $Y$ be a translation map. Let $O_{Y}$ denote the set of operators $Y(A)$. We know that $O_{Y}$ is a commutative algebra of linear operators on $A$ and that the map $O_{Y} \longrightarrow A, Y(a) \longrightarrow Y(a) \mathbf{1}$ is a linear isomorphism.

Definition 4.3 A creative operator algebra for the based space $(A, \mathbf{1})$ is a commutative subalgebra $O$ of $\operatorname{Lin}(A)$ such that the map $O \longrightarrow A, x \mapsto x \mathbf{1}$ is a linear isomorphism. We call this map the creative map associated to $O$. We write $C O(V, \mathbf{1})$ for the set of creative operator algebras for $(V, \mathbf{1})$.

It turns out that in the above definition, it is enough to require that the creative map is surjective.

If $O$ is a creative operator algebra, let $Y_{O}: A \longrightarrow O \subset \operatorname{Lin}(A)$ be the inverse of the creative map $O \longrightarrow A$ associated with $O$. Thus $Y_{O}(x \mathbf{1})=x$ for all $x \in O$, or equivalently $Y_{O}(a) \mathbf{1}=a$ for all $a \in A$. It is easy to check that $Y_{O}$ is a translation map.

Proposition 4.4 The sets $T(A, \mathbf{1})$ and $C O(A, \mathbf{1})$ are in bijective correspondence via the map $Y \mapsto O_{Y}$ and its inverse map $O \mapsto Y_{O}$. 
To gain further insight into creative operator algebras we state:

Proposition 4.5 If $O$ is in $C O(A, \mathbf{1})$, then

(i) $O$ is a maximal commutative subalgebra of $\operatorname{Lin}(A)$;

(ii) $O$ is a complementary subspace to the annihilator $A n n(\mathbf{1})$ of $\mathbf{1}$ in $\operatorname{Lin}(A)$.

\section{$5 \quad$ Vertex Operator Algebras}

Recall that the formal variable $z$ is assigned degrees $|a|=0,\|z\|=-1$.

Definition 5.1 国同 $A$ VOA is a doubly graded vector space $V=\oplus V^{k}[l]$ with a distinguished element 1 with both degrees equal to zero, and a degree preserving linear map $Y(-, z): V \longrightarrow Q O(V)$, satisfying the following conditions:

(i) (Boundedness) For each $k, V^{k}[l]=0$ for all but finitely many negative $l$;

(ii) (Unit) $Y(\mathbf{1}, z)=i d$;

(iii) (Nondegeneracy) For any $v$ in $V, Y(v, z) \mathbf{1}$ is a formal power series and

$$
\lim _{z \rightarrow 0} Y(v, z) \mathbf{1}=v .
$$

(iv) (Cauchy-Jacobi identity) For any $v, v^{\prime}$ in $V$, and integers $n, k, l$

$$
\begin{aligned}
& \operatorname{Res}_{z-w} Y\left(Y(v, z-w) v^{\prime}, w\right) \iota_{w, z-w} z^{n} w^{k}(z-w)^{l} \\
& =\operatorname{Res}_{z} Y(v, z) Y\left(v^{\prime}, w\right) \iota_{z, w} z^{n} w^{k}(z-w)^{l} \\
& -(-1)^{|v|\left|v^{\prime}\right|} \operatorname{Res}_{z} Y\left(v^{\prime}, z^{\prime}\right) Y(v, z) \iota_{w, z} z^{n} w^{k}(z-w)^{l}
\end{aligned}
$$

For now we will ignore the so-called Virasoro structure on a VOA. (See below.)

We want to develop the theory of VOA's as a straightforward but deep generalization of the theory of commutative algebras. We have developed three points of view about commutative algebras:

1. Bilinear product on a based space;

2. Translation map for a based space;

3. Creative operator algebra for a based space.

We have demonstrated the elementary equivalence of these three points of view. Let's sketch informally what we know about these three levels for VOA theory.

1. A VOA can be described in the following terms: 
(i) A bigraded vector space $V=\oplus V^{k}[l]$ with a distinguished element 1of degrees $|\mathbf{1}|=0=\|\mathbf{1}\|$. We will assume for simplicity that the second degree is bounded from below.

(ii) A family of bilinear products $m_{n}, n \in \mathbf{Z}$, on $V$. The degrees of $m_{n}$ are $\left|m_{n}\right|=0, \| m_{n}||=-n-1$. Thus for any pair of elements $a, b$ in $V, m_{n}(a, b)=0$ for all but finitely many positive $n$. Only $m_{-1}$ is of second degree zero.

(iii) Axioms relating to 1: for any $a$ in $V$,

a) for any nonnegative integer $n, m_{n}(a, \mathbf{1})=0$

b) $m_{-1}(a, \mathbf{1})=a$

c) for any integer $n \neq-1, m_{n}(\mathbf{1}, a)=0$

d) $m_{-1}(\mathbf{1}, a)=a$.

Thus the operation $m_{-1}$ behaves most classically: $\mathbf{1}$ is an identity element for $m_{-1}$. For the operation $m_{n}$ with $n<-1, \mathbf{1}$ is only a right identity. For the operation $m_{n}$ with $n>0, \mathbf{1}$ is a universal left and right zero divisor.

(iv) Cauchy-Jacobi axiom system: This is a complex system of trilinear identities for the system of operations $m_{n}$. Two kinds of terms appear: $m_{n}\left(a, m_{p}(b, c)\right)$, $m_{p}\left(m_{n}(a, b), c\right)$ with various permutations of the three elements of $V$.

Remark 5.2 (i) It would be very difficult at the present time to motivate the Cauchy-Jacobi axiom system in the purely classical setting of systems of bilinear products. For one thing, it would be difficult to give a simple and compelling example of an algebra satisfying all of the above axioms. Moreover, the actual known examples of VOA's did not arise in the language of this first level.

(ii) It is already difficult to motivate algebras with more than two bilinear products. We know that in some sense the operations $m_{n}$ in a VOA fall into two classes: $n$ nonnegative, and $n$ negative. We also know that in applications, the two operations, $m_{0}$ and $m_{-1}$ appear most often. However, the operation $m_{1}$ also occurs in our work on BV operators (see below). Thus the BV operation in this language is $m_{1}(b, v)$ where $b$ is a distinguished element.

2. Associated to a VOA $V$ is the vertex map, $Y_{m}$, where $m$ now stands for the sequence of operations $m_{n}$ in $V$ :

$$
Y_{m}(v, z) v^{\prime}=\sum_{n} m_{n}\left(v, v^{\prime}\right) z^{-n-1}
$$

To hide the direct reference to $m$, we can introduce the mode operators $v(n)$ such that $\|v(n)\|=\|v\|-n-1$ if $v$ is homogeneous, and such that

$$
m_{n}\left(v, v^{\prime}\right)=v(n) v^{\prime}
$$


Finally we introduce the vertex operator

$$
Y(v, z)=\sum_{n} v(n) z^{-n-1}
$$

for each $v$ in $V$. We can write the standard duality axioms for a VOA in terms of the vertex operators above. We obtain axioms that look like generalizations of the axioms for a translation map (Definition 4.1).

(i) $Y(\mathbf{1}, z)=i d$;

(ii) For any $v$ in $V, Y(v, z) \mathbf{1}$ is a formal power series and

$$
\lim _{z \rightarrow 0} Y(v, z) \mathbf{1}=v .
$$

(iii) For any $v, v^{\prime}$ in $V$ [5] [6],

$$
Y(v, z) Y\left(v^{\prime}, z^{\prime}\right) \sim(-1)^{|v|\left|v^{\prime}\right|} Y\left(v^{\prime}, z^{\prime}\right) Y(v, z) \sim Y\left(Y\left(v, z-z^{\prime}\right) v^{\prime}, z^{\prime}\right),
$$

where the relation $\sim$ has to be defined carefully in terms of the formal variable calculus. In particular we need to assume the rationality of the matrix entries of the three formal expressions above. (See Proposition 3.7.)

Observation: If all the vertex operators $Y(v, z)$ are constant ( $z$-independent) operators in $V$, then $Y_{m}$ is exactly a translation map in the sense of commutative algebra, and we can regard $V$ as a graded commutative algebra with operation $m_{-1}$.

3. We want to take the third perspective: for each $v$ in $V, Y(v, z)$ is a "quantum operator". We want to ask several questions about these quantum operators:

(i) What sort of properties does $Y(v, z)$ have as a quantum operator on its own? In other words, what class of quantum operators arises from the study of VOA's. (ii) What properties does the space of all vertex operators have as linear subspace of the space of all quantum operators?

We recall that $Q O=Q O(V)$ denotes the space of all quantum operators built from $\operatorname{Lin}(V)$. We write $Q O_{Y}=Q O(V, Y)$ as the space of all quantum operators of the form $Y(v, z), v$ in $V$, for a fixed VOA structure $Y$ on $V$.

Again we want to characterize in simple terms what sort of subspaces of $Q O$ can be of the form $Q O_{Y}$ for some VOA structure $Y$ on $V$. Eventually, we want to understand how to concretely construct examples of VOA's by first constructing an appropriate subspace of $Q O(V)$. We need an appropriate generalization of the notion of a creative operator algebra.

Recall that we have defined a sequence of bilinear operations $\circ_{n}$ in $Q O$. It is a consequence of the Cauchy-Jacobi identity that the following holds:

Proposition 5.3 Suppose $(V, \mathbf{1}, Y)$ is a $V O A$, and $v, v^{\prime}$ are in $V$. Then for any 
integer $n$,

$$
Y(v, z) \circ_{n} Y\left(v^{\prime}, z\right)=Y\left(v(n) v^{\prime}, z\right) .
$$

In particular, $Q O_{Y}$ is closed with respect to all of the operations ${ }^{\circ}$. Moreover for $v, v^{\prime}$ in $V$,

$$
Y(v, z) \circ_{n} Y\left(v^{\prime}, z\right)=0
$$

for all sufficiently large positive $n$. This implies that $Q O_{Y}$ is a local quantum operator algebra (See Definition 3.6). In particular, a vertex operator is mutually local with itself. This is a highly nontrivial property for a quantum operator.

Motivated by property 2(iii) of VOAs, we introduce the following:

Definition 5.4 A commutative quantum operator algebra $O$ is a local $Q O A$ such that for any $a(z), b(z)$ in $O$,

$$
a(z) b(w) \sim(-1)^{|a||b|} b(w) a(z)
$$

in the sense that the matrix entries for both sides represent the same rational function. $V$.

Let's now introduce a distinguished element, $\mathbf{1}$ in the doubly graded space

Definition 5.5 (cf. Definition 5.1) A creative $Q O A$ for $(V, \mathbf{1})$ is a commutative QOA, O, such that

(i) For every $a(z)$ in $O, a(z) \mathbf{1}$ is a series with only nonnegative powers of $z$.

(ii) The map $O \longrightarrow V$,

$$
a(z) \mapsto \lim _{z \rightarrow 0} a(z) \mathbf{1}
$$

is a linear isomorphism. This map is called the creative map associated with $O$.

The reader should verify that every $\operatorname{VOA},(V, \mathbf{1}, Y)$, defines a creative QOA, $Q O_{Y}$. We now give the converse, which is the main new result of this paper:

Theorem 5.6 Let $(V, \mathbf{1})$ be a based space and let $O$ be a creative $Q O A$ for $(V, \mathbf{1})$. Then $(V, \mathbf{1}, Y)$ is a VOA where the vertex map $Y$ is given by the inverse of the creative map associated to $O$.

This theorem reformulates the Cauchy-Jacobi axiom in VOA theory in terms of locality, commutativity and creativity in QOA theory. The reader should compare our approach to that theorem of Frenkel-Lepowsky-Meurman which reformulates the Cauchy-Jacobi axiom in terms of rationality, associativity and commutativity [5.

Question: Given a commutative QOA, $O$, can we construct a creative QOA? 
Proposition 5.7 Let $O$ be a commutative QOA on the space $V$. Then for each nonzero vector $\mathbf{1}$ of degrees $|\mathbf{1}|=0, \| \mathbf{1}||=0$, there is a canonical subquotient $O_{1}$ of $O$ which is a creative $Q O A$ for some based space.

To construct $O_{1}$, we proceed as follows: let $O^{\prime}$ be the subspace of quantum operators $a(z)$ in $O$ such that $a(z) \mathbf{1}$ has only nonnegative powers of $z$. Let $V^{\prime}$ be the image of the map $\chi: O^{\prime} \longrightarrow V, a(z) \mapsto \lim _{z \rightarrow 0} a(z) \mathbf{1}$. We show that $O^{\prime}$ is a closed with respective to all of the operations $\circ_{n}$, hence is a commutative QOA on $V$. In fact by direct computation, we have for any $a(z), b(z)$ in $O^{\prime}$, and for any integer $n$ :

$$
\lim _{z \rightarrow 0} a(z) \circ_{n} b(z) \mathbf{1}=a(n) b(-1) \mathbf{1} .
$$

This implies that the algebra $O^{\prime}$ acts in $V^{\prime}$ by restriction. This means that $O^{\prime}$ is now a commutative QOA for the based space $\left(V^{\prime}, \mathbf{1}\right)$.

Now let $I$ be the two sided ideal generated by ker $\chi$ in $O^{\prime}$. Consider the subspace $\chi(I)$ in $V^{\prime}$. If $a(z)$ is in $O^{\prime}$ and $b(z)$ in $I$, then $a(z) \circ_{n} b(z)$ is in $I$. It follows from eqn (5.20) that $a(n) b(-1) \mathbf{1}$ is in $\chi(I)$ for all $n$. Thus we have shown that the action of $O^{\prime}$ in $V^{\prime}$ stabilizes the subspace $\chi(I)$. It is now clear that $O^{\prime}$ acts in $V^{\prime} / \chi(I)$ in a natural way. Moreover, we have an induced isomorphism $\chi: O_{1}=O^{\prime} / I \longrightarrow V^{\prime} / \chi(I)$. This map defines a creative map associated to the commutative QOA $O_{\mathbf{1}}$ for the based space $\left(V^{\prime} / \chi(I), \mathbf{1}\right)$.

\section{$5.1 \quad$ Examples}

Let $\mathcal{C}$ be the Clifford algebra with the generators $b(n), c(n)(n \in \mathbf{Z})$ and the relations [8]

$$
\begin{aligned}
b(n) c(m)+c(m) b(n) & =\delta_{n,-m-1} \\
b(n) b(m)+b(m) b(n) & =0 \\
c(n) c(m)+c(m) c(n) & =0
\end{aligned}
$$

Let $\lambda$ be a fixed integer. The algebra $\mathcal{C}$ becomes $\mathbf{Z}$-bigraded if we define the degrees $|b(n)|=-|c(n)|=-1,\|b(n)\|=\lambda-n-1,\|c(n)\|=-\lambda-n$. Let $\bigwedge^{*}$ be the graded irreducible $\mathcal{C}{ }^{*}$-module with generator 1 and relations

$$
b(m) \mathbf{1}=c(m) \mathbf{1}=0, \quad m \geq 0
$$

Let $b(z), c(z)$ be the quantum operators

$$
\begin{aligned}
b(z) & =\sum_{m} b(m) z^{-m-1} \\
c(z) & =\sum_{m} c(m) z^{-m-1}
\end{aligned}
$$


Let $O=O(b, c)$ be the smallest QOA containing $b(z), c(z)$.

Lemma 5.8 The QOA $O(b, c)$ is spanned by the following quantum operators (see section 3):

$$
: \partial^{n_{1}} b(z) \cdots \partial^{n_{i}} b(z) \partial^{m_{1}} c(z) \cdots \partial^{m_{j}} c(z):
$$

with $n_{1}>\ldots>n_{i} \geq 0, m_{1}>\ldots>m_{j} \geq 0$.

Let's sketch a proof. If $A(z)$ is in $O$, then so is $A(z) \circ_{-2} 1=\partial A(z)$ (see eqn (3.10)). Thus $O$ contains all the derivatives of $b(z), c(z)$. Since $O$ is closed with respect to the Wick product, the iterated Wick products of the derivatives are also in $O$. So it is enough to show that those iterated Wick products are closed under every $\circ_{n}$, ie. if $A(z), B(z)$ are two such products then $A(z) \circ_{n} B(z)$ is a sum of those products. This can be shown by induction and by computing the operator product expansions in two ways: $A(z) B(w)$ and $B(w) A(z)$.

By calculating the operator product expansion, one will discover that in fact $O$ is commutative as a QOA. Let's consider the map $\chi: O \longrightarrow \wedge, a(z) \mapsto$ $\lim _{z \rightarrow 0} a(z) \mathbf{1}$. Using the quantum operator given in the above Lemma, we get

$$
\begin{aligned}
& \lim _{z \rightarrow 0}: \partial^{n_{1}} b(z) \cdots \partial^{n_{i}} b(z) \partial^{m_{1}} c(z) \cdots \partial^{m_{j}} c(z): \mathbf{1}=n_{1} ! \cdots n_{i} ! m_{1} ! \\
& \cdots m_{j} ! b\left(-n_{1}-1\right) \cdots b\left(-n_{i}-1\right) c\left(-m_{1}-1\right) \cdots c\left(-m_{j}-1\right) \mathbf{1}
\end{aligned}
$$

Since the vectors on the right hand side form a basis for the space $\bigwedge$, the map $\chi$ is necessarily an isomorphism. It follows that $O$ is a creative QOA with the associated creative map $\chi$. Thus by Theorem 5.6, $\left(\bigwedge^{*}, \mathbf{1}, \chi^{-1}\right)$ is a VOA.

We remarked earlier that the products $\circ_{n}$ in a QOA are in general neither associative nor commutative as bilinear operations. We can now give a good example to illustrate this. Let's rescale $c(z)$ by a parameter $\hbar$ (Planck's constant) so that we have

$$
b(n) c(m)+c(m) b(n)=\hbar \delta_{n,-m-1} .
$$

It is clear that with this new relation the above construction still goes through almost word for word, and we obtain a creative QOA $O_{\hbar}$. Consider the following computations:

$$
\begin{aligned}
& :(: b(z) c(z):) b(z):-: b(z)(: c(z) b(z):)=\hbar \partial b(z) \\
& :(: b(z) c(z):) b(z):-: b(z)(: b(z) c(z):)=\hbar \partial b(z) .
\end{aligned}
$$

The first eqn shows that the Wick product (ie. $o_{-1}$ ) is nonassociative for $\hbar \neq 0$. The second eqn shows that it is noncommutative. In fact the obstruction for associativity and commutativity is of order $\hbar$. In this sense, $O_{\hbar}$ is a nonassociative deformation of the commutative associative algebra $O_{0}$. 
We now discuss our second example. Let $\mathfrak{g}$ be a finite dimensional simple Lie algebra with the standard invariant bilinear form $B$. Let $\hat{\mathfrak{g}}$ be the associated affine Kac-Moody Lie algebra whose bracket is given by

$$
[X(n), Y(m)]=[X, Y](n+m)+n \zeta B(X, Y) \delta_{n+m, 0}
$$

where $\zeta$ is a nonzero element of the 1 dimensional center of $\hat{\mathfrak{g}}$. This Lie algebra can be given a $\mathbf{Z}$ grading by $|X(n)|=0,\|X(n)\|=-n$ for all $X \in \mathfrak{g}$.

Let $k$ be a complex parameter and $L(\mathfrak{g}, k)$ be the graded irreducible $\hat{\mathfrak{g}}$-module generated by $\mathbf{1}$, with the relations:

$$
X(n) \mathbf{1}=0, \quad n \geq 0, X \in \mathfrak{g}, \quad \zeta \mathbf{1}=k \mathbf{1} .
$$

For each $X$ in $\mathfrak{g}$, define the quantum operator

$$
X(z)=\sum_{n} X(n) z^{-n-1} .
$$

This has degrees $|X|=0,\|X\|=1$. Let $O=O(\mathfrak{g}, k)$ be the smallest QOA in $Q O(L(\mathfrak{g}, k))$ such that $O$ contains all the $X(z)$.

Proposition $5.9 O(\mathfrak{g}, k)$ is a creative QOA. If $\left\{X_{1}, X_{2}, \ldots\right\}$ is a basis for $\mathfrak{g}$, then $O(\mathfrak{g}, k)$ is spanned by

$$
: \partial^{n_{11}} X_{1}(z) \partial^{n_{12}} X_{1}(z) \cdots \partial^{n_{21}} X_{2}(z) \partial^{n_{22}} X_{2}(z) \cdots \text { : }
$$

where $n_{11} \geq n_{12} \ldots, n_{21} \geq n_{22} \ldots, \ldots$

The proof is similar to the first example above.

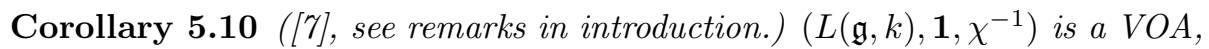
where $\chi$ is the creative map associated to $O(\mathfrak{g}, k)$.

\section{Main Theorems}

We now discuss the main results, which draw from all the notions introduced in this paper. We will also illustrate the results with some examples which are built out of the previous ones.

Let $O$ be a creative QOA. A quantum operator $L(z)$ is called Virasoro if for some constant $\kappa$ (called the central charge of $L$ ) [1],

$$
\begin{aligned}
L(z) L(w) & =\frac{\kappa}{2}(z-w)^{-4}+2 L(w)(z-w)^{-2}+\partial L(w)(z-w)^{-1}+: L(z) L(w): \\
L(z) a(w) & =\cdots+\|a\| a(w)(z-w)^{-2}+\partial a(w)(z-w)^{-1}+: L(z) a(w): \quad(6.30)
\end{aligned}
$$


for all homogeneous $a(z)$ in $O$. Here "..." denotes the higher order polar terms.

Definition 6.1 A pair $(O, L)$ is called a conformal $Q O A$ if $O$ is a creative $Q O A$ with a distinguished Virasoro quantum operator $L(z)$.

For example in the QOA $O(b, c)$ introduced above, for a fixed $\lambda$, we have a Virasoro quantum operator [8]

$$
L^{b c}(z)=(\lambda-1): c(z) \partial b(z)+\lambda: \partial c(z) b(z):
$$

with central charge $\kappa=-12 \lambda^{2}+12 \lambda-2$.

In the second example $O(\mathfrak{g}, k)$, if $k+h^{\vee} \neq 0$ we define (see [13])

$$
L^{\mathfrak{g}}(z)=\frac{1}{2\left(k+h^{\vee}\right)} \sum_{i}: X_{i}(z) X_{i}(z):
$$

where $h^{\vee}$ is the dual Coxeter number of $\mathfrak{g}$ and the $X_{i}$ is an orthonormal basis relative to the form $B$. It is a fundamental fact the $L^{\mathfrak{g}}(z)$ is a Virasoro quantum operator.

\subsection{The BRST construction}

It is evident that if $(O, L),\left(O^{\prime}, L^{\prime}\right)$ are conformal QOAs on the respective based spaces $(V, \mathbf{1}),\left(V^{\prime}, \mathbf{1}^{\prime}\right)$ with central charges $\kappa, \kappa^{\prime}$, then $\left(O \otimes O^{\prime}, L+L^{\prime}\right)$ is a conformal QOA on $\left(V \otimes V^{\prime}, \mathbf{1} \otimes \mathbf{1}\right)$ with central charge $\kappa+\kappa^{\prime}$. From now on we fix $\lambda=2$ which means that $L^{b c}$ now has central charge -26. Let $(O, L)$ be any conformal QOA with central charge $\kappa$ and consider

$$
C^{*}(O)=O^{*}(b, c) \otimes O .
$$

This QOA has a natural quantum operator called the BRST current [8] [3] [4]:

$$
J(z)=: c(z)\left(L(z)+\frac{1}{2} L^{b c}(z)\right): .
$$

which has degrees $|J|=|| J||=1$.

Lemma 6.2 14] Let $Q=\operatorname{Res}_{z} J(z)$. Then $Q^{2}=0$ iff $\kappa=26$.

Recall that for any element $a(z)$ in the QOA $C^{*}(O)$, we have $J(z) \circ_{0} a(z)=$ $[Q, a(z)]$. Thus the graded commutator $[Q,-]$ is a derivation of the QOA $C^{*}(O)$. For $\kappa=26,[Q,-]$ is a differential on the QOA $C^{*}(O)$ and we have a cochain complex

$$
[Q,-]: C^{*}(O) \longrightarrow C^{*+1}(O)
$$


It is called the BRST complex associated to $O$. Its cohomology will be denoted as $H^{*}(O)$. All the operations $\circ_{n}$ on $C^{*}(O)$ descend to the cohomology. However, all but one is trivial.

Theorem 6.3 24] 20 The Wick product $\circ_{-1}$ induces a graded commutative product on $H^{*}(O)$ with unit element represented by the identity operator. Moreover, every cohomology class is represented by a quantum operator a $(z)$ with $\|a\|=0$. $a(z)$.

Consider now the linear operator $\Delta: C^{*}(O) \longrightarrow C^{*-1}(O), a(z) \mapsto b(z) \circ_{1}$

Theorem 6.4 19 The operator $\Delta$ descends to the cohomology $H^{*}(O)$. Morever, it is a $B V$ operator on the commutative algebra $H^{*}(O)$. Thus $H^{*}(O)$ is naturally a $B V$ algebra.

The two main theorems above were originally proved in 19$]$ in the language of vertex operator algebras. It is Theorem 5.6 which allows us to translate between the two versions. (For related versions of Theorem 6.4, see [1] [22] 15] 12].)

Examples: Recall that $O(\mathfrak{g}, k)$ is a conformal QOA with a Virasoro quantum operator $L^{\mathfrak{g}}(z)$. Its central charge is $\kappa=\frac{k \operatorname{dim} \mathfrak{g}}{k+h^{\vee}}$. Since the dimension of a simple Lie algebra $\mathfrak{g}$ is never 26 , we can choose $k$ so that $\kappa=26$. With this choice, we can consider the BRST cohomology $H^{*}=H^{*}(O(\mathfrak{g}, k))$. The answer is given as follows:

$$
\begin{aligned}
H^{0} \cong \mathbf{C} \\
H^{1} \cong \mathfrak{g} \\
H^{2} \cong \mathfrak{g} \\
H^{3} \cong \mathbf{C}
\end{aligned}
$$

Recall that given a Lie algebra, its exterior algebra is a BV algebra. As a BV algebra, the structure of $H^{*}$ is determined by the following:

Proposition 6.5 There is a unique surjective homomorphism of BV algebras $\bigwedge^{*} \mathfrak{g} \longrightarrow H^{*}(O(\mathfrak{g}, k))$ such that under the above identification (6.36), $X \mapsto$ $X \in H^{1}$, for all $X$ in $\mathfrak{g}$.

\section{References}


[1] A. Belavin, A.M. Polyakov and A.A. Zamolodchikov, "Infinite conformal symmetry in two dimensional quantum field theory", Nucl. Phys. B241 (1984) 333.

[2] R.E. Borcherds, "Vertex operator algebras, Kac-Moody algebras and the Monster", Proc. Natl. Acad. Sci. USA. 83 (1986) 3068.

[3] B. Feigin, "Semi-infinite homology for Virasoro and Kac-Moody algebras", Usp. Mat. Nauk. 39 (1984) 195-196.

[4] I.B. Frenkel, H. Garland and G.J. Zuckerman, "Semi-infinite cohomology and string theory", Proc. Nat. Acad. Sci. U.S.A. 83 (1986) 8442.

[5] I.B. Frenkel, J. Lepowsky and A. Meurman, "Vertex Operator Algebras and the Monster", Academic Press, New York, 1988.

[6] I.B. Frenkel, Y. Huang and J. Lepowsky, "On axiomatic approaches to vertex operator algebras and modules", Yale-Rutgers preprint, Dept. of Math., 1989.

[7] I.B. Frenkel and Y. Zhu, "Vertex Operator Algebras associated to affine Lie algebras and the Virasoro algebra", Duke Math J. 66 (1992) 123.

[8] D. Friedan, E. Martinec and S. Shenker, "Conformal invariance, supersymmetry and string theory", Nucl. Phys. B271 (1986) 93.

[9] M. Gerstenhaber, "The cohomology structure of an associative ring", Ann. of Math. 78, No.2 (1962) 267.

[10] M. Gerstenhaber, "On the deformation of rings and algebras", Ann. of Math. 79, No.1 (1964) 59.

[11] E. Getzler, "Batalin-Vilkovisky algebras and two dimensional topological field theory", MIT preprint, hepth/9212043.

[12] Y-Z. Huang, "Operadic formulation of topological vertex algebras and Gerstenhaber or Batalin-Vilkovisky algebras", Univ of Penn preprint, hepth/9306021.

[13] V. Kac and A. Raina, Highest weight representations of infinite dimensional Lie algebras, Advanced Series in Math Physics, Vol. 2, World Scientific (1987). 
[14] M. Kato and K. Ogawa, "Covariant quantization of string based on BRS invariance", Nucl. Phys. B212 (1983) 443.

[15] T. Kimura, J. Stasheff and A. Voronov, "On operad structures of moduli spaces and string theory", UNC preprint, hepth/9307114.

[16] B.H. Lian and G.J. Zuckerman, "New selection rules and physical states in 2d gravity; conformal gauge", Phys. Lett B254, No.3,4 (1991) 417.

[17] B.H. Lian and G.J. Zuckerman, "2d gravity with $\mathrm{c}=1$ matter",Phys. Lett. B266 (1991) 21.

[18] B.H. Lian and G.J. Zuckerman, "Semi-infinite homology and 2D gravity (I)", Commun. Math. Phys. 145 (1992) 561.

[19] B.H. Lian and G.J. Zuckerman, "New perspectives on the BRST-algebraic structure in string theory", hepth/9211072, Commun. Math. Phys. 154 (1993) 613.

[20] G. Moore, "Finite in All Directions", Yale preprint, hepth/9305139.

[21] M. Penkava, "A note on BV algebras", UC Davis preprint November 92.

[22] M. Penkava and A. Schwarz, "On some algebraic structures arising in string theory", UC Davis preprint, hepth/9212072.

[23] A. Schwarz, "Geometry of Batalin-Vilkovisky quantization", UC Davis preprint, hepth/9205088.

[24] E. Witten, "Ground ring of the two dimensional string theory", hepth/9108004, Nucl. Phys. B373 (1992) 187.

[25] E. Witten, "A note on the anti-bracket formalism", preprint IASSNS-HEP$90 / 9$.

[26] E. Witten and B. Zwiebach, "Algebraic structures and differential geometry in 2d string theory", hepth/9201056, Nucl. Phys. B377 (1992) 55.

DEPARTMENT OF MATHEMATICS, HARVARD UNIVERSITY CAMBRIDGE, MA 02138. lian@math.harvard.edu

DEPARTMENT OF MATHEMATICS, YALE UNIVERSITY NEW HAVEN, CT 06520. gregg@math.yale.edu 